\title{
Presenting a Medical Expert System for Diagnosis and Treatment of Nephrolithiasis
}

\author{
Mirpouya Mirmozaffari*
}

\begin{abstract}
Expert systems aim to employ expert skills for non-expert person. These designs simulate intellectual and practical performance of human making the performance of expert systems close to that of an expert human. Various expert systems have been proposed in medicine, thus this area is attracting attention. Most problems in modern medicine are very complicated and there is no logic reason for accurate decision making. To this end, doctors decide arbitrarily and variably. On the other hand, large volume of medical information makes decision making more difficult while modern technologies add to volume of information and make problems more difficult. Considering these problems, there is a great challenge in medical diagnosis which requires decision-making support systems. In this paper, an expert system is presented for diagnosis and treatment of nephrolithiasis in which knowledge required for diagnosis and treatment is stored as rules in the system knowledge base. If experts are absent, diagnosis and treatment can be done reliably. Expert systems can be used as decision support by the users. However, currently, they cannot replace experts.
\end{abstract}

Index Terms-Expert systems, medical decision-making support systems, nephrolithiasis

\section{INTRODUCTION}

Most problems in modern medicine are very complicated and there are no compelling reasons for accurate decisionmakings. Doctors usually decide arbitrarily and variably. On the other hand, volume of medical information related to a small diagnostic part is so large that fast and accurate decision-making is difficult and complex. In addition, modern technologies increase volume of this information and make the problems even more complicated. In fact, doctors have never faces such volume of information. Furthermore, people require specific decisions, because their features are different from what is mentioned in medical references and each one has its own unique requirements. Considering the mentioned problems, it can be easily understood that there is a great challenge in medical diagnosis which requires decision support systems.

Decision support systems in medicine are referred to computer software which are built to aid clinical diagnosis. These systems employ medical information and knowledge to diagnose various diseases and offer medical suggestions to the patients. In fact, they have not been designed to replace doctors but they aim to aid medical experts to diagnose diseases based on a series of empirical rules.

*Corresponding Author:

PhD student, Gianforte School of Computing, Montana State University,

Bozeman, Montana, USA. (m.mirmozaffari@gmail.com)

DOI: http://dx.doi.org/10.24018/ejmed.2019.1.1.20

\section{LITERATURE REVIEW}

History of medical decision support systems is real history of mutual cooperation among doctors and mathematicians. As electronic computers emerged in 1950 to 1960 , first medical decision support systems were innovated with different healthcare purposes. It was announced that doctors are not usually aware how to solve diagnostic problems. It was also claimed that in order to model diagnosis process, both logic rules (sets theory and Boolean algebra) and inference calculations (Bayes rules) are required. In 1961, Warner et.al designed one of the first medical decision support systems based on Bayes rules. Indeed, the first system, was a system designed by de Dombal to diagnose acute abdominal pain and it was employed in many healthcare centers. In 1965, DENDRAL was presented to describe molecular structure. In 1976, MYCIN was presented to diagnose vascular diseases. PUFF was also proposed to diagnose pulmonary diseases. XBONE was also designed to diagnose bone diseases. VM was designed to monitor patients requiring intensive care. CADCUCEUS was also presented to diagnose internal diseases, BLUE BOX was proposed to diagnose and treat depression and expert systems for diagnosing acid material and electrolytes, anesthetic management education and diagnosing internal diseases are all among such systems [1].

Fuzzy and expert systems technologies provide a number of advantages to the science and engineering fields. Fuzzy systems store knowledge of experts in a form of rules or mathematical expressions that is easy to visualize, enter into the system, and modify. Also, membership functions and parameters are incorporated so that they allow the system to achieve a good performance [2]. Several methods have been proposed in the literature to improve the performance of fuzzy logic systems [3-5]. In [6], the fuzzy system has been used for developing a control scheme for hydropower generators in water distribution systems. This method was successfully able to control the operation of the generator for obtaining the maximum efficiency. In expert systems, logical operations are implemented in a form that is relatively easy to comprehend. In [7], the authors propose an expert system using an intelligent softbots and prepare a portfolio of IS that has the maximum alignment with the strategy plan. In [8], the author developed an expert system using the VP-Expert shell for identifying liver illnesses. Assessing other fatal disease such as heart disease plays a significant role as well. In so doing, Data Envelopment Analysis (DEA) offers a relative efficiency for each Decision Making Units (DMU) with several inputs and outputs [9]. Despite of healthcare Fuzzy 
expert system and data mining play an important roles in other fields of study such as using decision tree for ecoefficiency evaluation [10]. This paper is to presents an expert system for diagnosing and treating nephrolithiasis. In the following, the problem is described and then kidney stone is explained. Finally, a practical example is used to describe performance of a designed system. An expert medical system is a computer program. If they are implemented correctly, they help in efficient diagnosis of diseases and their treatment. Diagnosis and predicting side effects are performed after reception of patient information. This information is usually exchanged between patient and the doctor. Medical expert systems have features which discriminate them from other medical software. One of such differences is that systems mimic inferences of a doctor stepby-step to make correct results. In most cases, a system expert is aware of sequential inferences. It is clear that these expert systems require a large number of rules and medical facts to offer accurate results.

This paper aim to employ a software to achieve all advantages of an expert system to diagnose and treat nephrolithiasis.

\section{SPECIAL TERMS}

\section{A. Kidney}

Kidneys are organs of the body which their function is essential for life. Most people are born with two kidneys in the middle of the lumbar where each one is at one side of the vertebral column. Kidneys are related to urine. As urine is produces, kidneys excrete additional water and salt. Kidneys are chemical masters of the body and perform various functions described in the following.

\section{B. Body Liquids}

Kidneys excrete and preserve body liquids. If someone consumed a large amount of salt in his/her diet, kidneys excrete additional water and salt while if kidneys malfunction, occlusion of additional water and salt result in edema of tissues and limbs like hands, feet and around eyes.

\section{Excretion of Chemical Material of the Body}

Kidneys preserve required chemical materials of the body. Potassium is one of the materials required for muscular actions. If kidneys do not operate correctly, amount of potassium becomes unbalances resulting in muscular weakness.

\section{Excretion of Additional Material from Body}

Additional material in the body is created through breakage of food protein and usual muscular activities. Kidneys excrete such material which is known as urea. Inefficiency of kidneys in excretion of this material results in uremia.

\section{E. Hormone Secretion}

Hormones are combinations secreted by glands so that another organ is activated to perform a specific action. These hormones help controlling blood pressure; if kidney dysfunctions, blood pressure oscillates due to defective secretion of these hormones.

\section{F. Nephrolithiasis}

One of the main factors resulting in kidney dysfunction is nephrolithiasis. Calcium and uric acid are the main types of kidney stones.

Calcium oxalate and calcium phosphate comprise about $75 \%$ to $85 \%$ of all kidney stones. Since stone grows on the surface of carbuncles or urine collecting pipes of kidneys and create no symptoms. However, in many cases abdominal radiographies performed on the abdomen for other reasons are discovered.

But what is important in prevention before treatment? There are many factors which cause nephrolithiasis among which excess consumption of animal protein, drinking grapefruit juice, lemon juice, salt, obesity, and sedentary life can be mentioned. There are other factors like genetic factors which cause nephrolithiasis.

Knowing the factors which result in nephrolithiasis, it can be treated depending on the type of stone. Fortunately, most stones are excreted without surgery but many of them are not excreted on their own and require pharmacotherapy. About $90 \%$ of stones can be excreted through consumption of liquids.

Surgery is required when stones:

1. Are large or growing.

2. They are not excreted after the expected time.

3 . Pain is permanent and stable.

4. Stone prevents urine excretion.

5 . There exists urine infection.

6. Kidney tissue is destructed.

Finally, if it results in kidney fluctuations, kidney should be dialyzed and if it does not get better, kidney transplant is the last solution.

\section{METHODOLOGY}

In this paper, VP-EXPERT is used to design an expert system for medical decision support to diagnose diseases and offer treatment solutions to patients suffering from nephrolithiasis. Expert systems are used in medicine due to the following:

1. People expertise is temporary but computer expertise is permanent.

2. Experts might be on their vacations which affects their performance. But Computers are not affected by these factors.

3. Local transmission of expertise is difficult but accessing software sis easier.

4. Expertise is usually more expensive than software with the same result.

And other advantages like high performance, high speed, reliability, flexibility, reducing dangers, stability and multiple expertise.

To access an expert system in order to treat nephrolithiasis, designing a knowledge base is necessary. There are three main sections in a knowledge base as follows: 
1. ACTIONS: including a set of commands which should be performed by the software. In fact, commands of interest are written in this block.

2. RULE: including a set of rules based on which commands are executed.

3. STATEMENTS: it is a set through which the user interacts with the program and exchange its information.

In order to complete these sections, an expert nephrolithiasis program is required. Mockler diagram associated to nephrolithiasis diagnosis is shown in Fig 1.

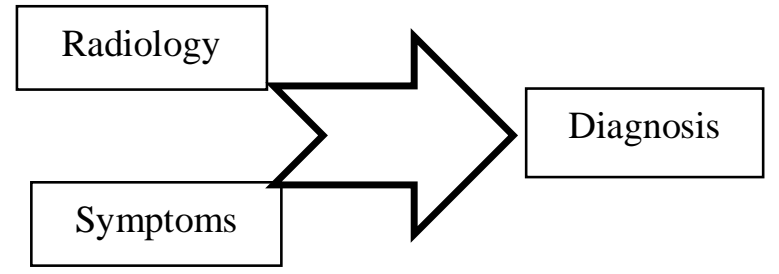

Fig.1. Mockler diagnosis diagram

\section{DESIGNING THE KNOWLEDGE BASE}

In order to design the knowledge base, first, ACTIONS section of the base and other section should be completed which are described in the following:

\section{A. Actions}

This section includes commands required for implementing the program. In this part, it is tried to explain the required variables to the user so that the user does not face problems while executing the programs. The program is commanded to find a specific variable to offer a treatment to the patient while execution.

\section{B. Rule}

This section is one of the most important sections of a knowledge base because it contains rules. As mentioned, treatment of nephrolithiasis at its first stages requires drinking liquids and exercising; then, if it is not treated, medicine should be taken along with drinking water and exercising. Final stage is to perform lithotrity, surgery, dialysis, and kidney transplant. Facts and rules of the knowledge base are designed accordingly. Systems ask the user to diagnose disease symptoms and suggest required commands.

Among disadvantages of expert systems, inability to detect validity of inputs by the user can be mentioned.

\section{Statement}

In this section, some questions are asked based on which rules and facts are investigated by the system and final result is given to the user. It can respond with respect to the options offered by the system.

\section{INFERENCE MOTOR}

In systems based on rules, inference motor chooses a rule for test to see if its conditions are correct or no? These conditions might be investigated through asking the user or through facts obtained through interview. When conditions of a rule are correct, its result would be correct. So, this rule is activated and its result is added to the knowledge base. Since
VP-EXPERT is used in this system and it does not require inference motor, designing the expert system would be easy. User interface of an expert system should have high exchange capacity so that information exchanged is done as conversation of a requestor and an expert. These steps are described using an example.

\section{IMPLEMENTATION}

Consider a person who suddenly sees edema in his/her feet or hands, or lose his/her blood pressure balance and he/she is diagnosed with nephrolithiasis. This person aims to diagnose his/her disease and find a way to treat it using an expert system. After diagnosing the disease, system offers a treatment solution depending on condition of the patient. In order to understand how the system offers a treatment solution, program implementation is suggested.

\section{CONCLUSION}

In this paper, an expert system is presented to diagnose and treat nephrolithiasis. Primary precaution clinicians need to be attentive to the probability of nephrolithiasis and its consequences to decide upon a diagnostic method. In so doing, objectives of the expert system were defined first. Then, previous studied were reviewed and expert system environment was described. In the next step, the system of interest is analyzed. Finally, the expert system which can support medical decisions is executed. It should be noted that systems should be presented which can simulate behavior of experts which is not always possible.

\section{REFERENCES}

[1] M. Ghazanfari, and Z Kazemi, Expert Systems. Tehran: Elmo Sanat; 2004.

[2] L. A. Zadeh,"The concept of a linguistic variable and its application to approximate reasoning-1," Information Sciences, vol. 8, pp. 199-249, 1975.

[3] D. Hidalgo, O. Castillo, and P. Melin, "Type-1 and type-2 fuzzy inference systems as integration methods in modular neural networks for multimodal biometry and its optimization with genetic algorithms," Information Sciences, vol. 179, no. 13, pp. 2123-2145, 2009.

[4] P. Melin, O. Mendoza, and O. Castillo, "Face recognition with an improved interval type-2 fuzzy logic sugeno integral and modular neural networks," IEEE Transactions on systems, man, and cybernetics-Part A: systems and humans, vol. 41, no. 5, pp. 1001-1012, 2011.

[5] H. B. Mitchell, "Pattern recognition using type-II fuzzy sets," Information Sciences, vol. 170, no. 2-4, pp. 409-418, 2005.

[6] O. A. Gashteroodkhani, M. Majidi, M. Etezadi-Amoli, "A Fuzzy-based Control Scheme for Recapturing Waste Energy in Water Pressure Reducing Valves" IEEE Power and Energy Society General Meeting (PESGM), pp. 1-5, Portland, OR, Aug 2018.

[7] MF. Zarandi, M. Tarimoradi, MA. Shirazi, IB Turksan. Fuzzy intelligent agent-based expert system to keep information systems aligned with the strategy plans: A novel approach toward SISP. Annual Conference of the North American Fuzzy Information Processing Society (NAFIPS) held jointly with 2015 th World Conference on Soft Computing (WConSC) 2015 Aug 17 (pp. 1-5). IEEE.

[8] M. Mirmozaffari, "Developing an Expert System for Diagnosing Liver Diseases”, EJERS, vol. 4, no. 3, pp. 1-5, Mar. 2019.

[9] M.Mirmozaffari and A. Alinezhad, "Ranking of Heart Hospital s Using cross-efficiency and two-stage DEA," $2017 \quad 7^{\text {th }}$ International Conference on Computer and Knowledge Engineering (ICCKE), Mashhad, 2017, pp. 217-222

[10] M. Mirmozaffari, "Eco-Efficiency Evaluation in Two-Stage Network Structure: Case Study: Cement Companies". Iranian Journal of Optimization (IJO). Dec. 16, 2018. 